Acta Protozool. (2017) 56: 181-189

www.ejournals.eu/Acta-Protozoologica

doi:10.4467/16890027AP.17.016.7497

PROTOZOOLOGICA

\title{
Allovahlkampfia minuta nov. sp., (Acrasidae, Heterolobosea, Excavata) a New Soil Amoeba at the Boundary of the Acrasid Cellular Slime Moulds
}

\author{
Alvaro DE OBESO FERNADEZ DEL VALLE, Sutherland K. MACIVER
}

Biomedical Sciences, Edinburgh Medical School, University of Edinburgh, Scotland, UK

Abstract. We report the isolation of a new species of Allovahlkampfia, a small cyst-forming heterolobosean soil amoeba. Phylogenetic analysis of the 18S rDNA and the internal transcribed spacers indicates that Allovahlkampfia is more closely related to the acrasids than to other heterolobosean groups and indicates that the new strain (GF1) groups with Allovahlkampfia tibetiensis and A. nederlandiensis despite being significantly smaller than these and any other described Allovahlkampfia species. GF1 forms aggregated cyst masses similar to the early stages of Acrasis sorocarp development, in agreement with the view that it shares ancestry with the acrasids. Time-lapse video microscopy reveals that trophozoites are attracted to individuals that have already begun to encyst or that have formed cysts. Although some members of the genus are known to be pathogenic the strain GF1 does not grow above $28^{\circ} \mathrm{C}$ nor at elevated osmotic conditions, indicating that it is unlikely to be a pathogen.

\section{INTRODUCTION}

The class heterolobosea was first created on morphological grounds to unite the schizopyrenid amoebae/amoeboflagellates with the acrasid slime moulds (Page and Blanton 1985), and subsequent molecular genetic data supported this union (Roger et al. 1996; Keeling and Doolittle 1996). The locomotory habit of these amoebae is to tend to produce an eruptive pseudopod, often alternatively from one side and then the other, of the advancing front. The heterolobosea are not directly related to other amoebae such as those within the Amoebozoa, despite being similar in appearance

Address for correspondence: Sutherland K. Maciver, Biomedical Sciences, Edinburgh Medical School, University of Edinburgh, Hugh Robson Building, George Square. Edinburgh. EH8 9XD, Scotland, UK; Phone: 44(0)131650 3714; E-mail: smaciver@ed.ac.uk and habit. The heterolobosean acrasid slime moulds are very similar to the amoebozoan slime moulds too in life cycle, but these remarkable similarities in appearance and function are most probably due to parallel evolution.

It is not usually possible to classify the trophozoites of this group morphologically since they share very similar habits and appearances but many heterolobosean amoebae are also capable of transforming into flagellates, these are so called amoeboflagellates. In addition, some heterolobosean groups are comprised of flagellates only (e.g. Percolomonas and Stephanopogon) having apparently lost the ability to adopt the ancestral amoeboid habit. Heterolobosean amoebae which do not form flagellates were traditionally placed in the genus Vahlkampfia (Page 1976) but it has since been shown that this group is not monophyletic and other genera such as Paravahlkampfia and Neovahlkampfia were 
formed (Brown and De Jonckheere 1999), to join other non-flagellate genera raised on morphological grounds. Further analysis of 18S RNA genes has allowed many other organisms to be included with the Heterolobosea and it is increasingly clear that it is an order with huge diversity (Pánek and Čepička 2016; Pánek et al. 2017). In 2009 it was necessary to raise a further genus, named Allovahlkampfia to place a new heterolobosean that did not fit elsewhere (Walochnik and Mulec 2009). A number of other isolates have now been added and as the genus Allovahlkampfia is presently configured, does not contain flagellates or amoeboflagellates but all members so far characterised, produce cysts without opercula. Members of Allovahlkampfia vary significantly in SSU rRNA sequence, with inserts present in some (Geisen et al. 2015). They are variable in size but the strain described in the present study which we name Allovahlkampfia minuta, is the smallest.

One heterolobosean amoeboflagellate, Naegleria fowleri is dangerously pathogenic for humans (Carter 1970; Siddiqui et al. 2016). Additionally, Allovahlkampfia spelaea is reported to infect the human eye (Huseein et al. 2016; Tolba et al. 2016) and to host bacteria known to be human pathogens (Mohamed et al. 2016) making this genus particularly worthy of study.

\section{METHODS AND MATERIALS}

Soil samples were collected from the Glenfinnan viaduct at the north end of Loch Shiel, Scotland, UK. Small quantities were settled on non-nutrient agar overlain with Neff's saline overnight. Strips of this were then inverted onto non-nutrient agar plates spread with E. coli (BL21) and monitored over a week for amoebal out growth. Small blocks were cut from the leading outgrowth onto fresh agar plates spread with $E$. coli until clones of strain GF1 were established. Large quantities of cloned GF1 amoebae were cultured on $2 \%$ agar YME plates containing $0.01 \%$ yeast extract and $0.025 \%$ malt extract overlain with Neff's saline at room temperature with E. coli as food organisms. For long term storage, the amoebae and cysts used in this study were taken up in Neff's saline with $10 \%$ DMSO and placed overnight in a freezer at $-20^{\circ} \mathrm{C}$, then transferred to $-80^{\circ} \mathrm{C}$ freezer. Amoebae were also stored long term in clay pellets as previously described (Lorenzo-Morales and Maciver 2006).

To test tolerance to osmotic stress, amoebae were seeded on E. coli spread non-nutrient agar plates supplemented with $0.5 \mathrm{M}$, $1 \mathrm{M}$ and 1.5 M mannitol as previously described (Khan et al. 2001). Temperature tolerance was also tested by seeding amoebae on E. coli spread non-nutrient agar plates incubated at various temperature and monitored for growth over a ten day period.

We investigated the ability of $A$. minuta to develop a flagellate stage with a variety of stimuli included the addition of distilled water, rapid changes in $\mathrm{pH}, \mathrm{CO}_{2}$ and $\mathrm{O}_{2}$ levels in addition to physical agitation (Perkins and Jahn 1970).
DNA was isolated from GF1 as previously described (Lorenzo-Morales et al. 2005). Amoebae were lysed in buffer and treated with proteinase $\mathrm{K}$ at $60^{\circ} \mathrm{C}$ for 2 hours followed by phenol-chloroform extraction. DNA was then concentrated by precipitation with isopropanol. The resulting DNA was quantified using a "nanodrop" spectrophotometer (ThermoFisher). An aliquot of $100 \mathrm{ng}$ of genomic DNA was used per PCR reaction, using GoTaq Green Master Mix polymerase (Promega).

Sequences were obtained from GenBank and other sources and compiled together with the new sequences from this study using "Seaview", version 4 (Gouy et al. 2010). "BioEdit" (Hall 1999) was used to trim sequences and to determine levels of homology between sequences. Sequences were aligned using seaview 4 which was also used to implement the PhyML algorithm to produce Maximum likelihood phylogenetic trees (Guindon and Gascuel 2003) using the GTR model. The non-parametric analysis was performed with 100 bootstrap pseudo-replicates, using representative sequences from other heterolobozoans (Naegleria, Tetramitus and Vahlkampfia) as the outgroup. Binomial names of the strains described previously (Geisen et al. 2015) were obtained from the CCAP website (https:// www.ccap.ac.uk).

\section{RESULTS}

The locomotive form is noticeably smaller than that of previously described members of this genus (Table 1). It is often wider than it is long although this is usually temporary. Pseudopods are produced eruptively from the anterior in a manner typical of the heterolobosean amoebae (Fig. 1). The trophozoites can also adopt a flabellate-like morphology. In culture GF1 trophozoites and cysts were observed to collect at the water air boundary and migrated on the meniscus. Strain GF1 was found to produce aggregates of cysts on agar plates (Fig. 2). Time-lapse video microscopy (see supplementary data and Fig. 3) reveals that the aggregated nature of the cyst distribution results from trophozoites actively migrating toward preformed cysts and not as a result of cyst aggregating by a passive mechanism after formation. GF1 was found to grow at $4{ }^{\circ} \mathrm{C}$ and slowly at $28^{\circ} \mathrm{C}$ but at $30^{\circ} \mathrm{C}$ it encysts. The optimal temperature for growth was $24^{\circ} \mathrm{C}$. GF1 could not grow on plate supplemented with $0.5 \mathrm{M}$ mannitol or above.

Phylogenetic analysis tentatively suggests that genus Allovahlkampfia is comprised of eight or nine groups, probably representing as many natural species. More data from more genes and isolates are required to clarify this of course. No flagellated cells have been found in culture despite the application of a range of previously reported flagellate inducing stimuli. Strain GF1 is closely related to two previously reported species (A. nederlandiensis and A. tibetiensis) (Fig. 4A) 


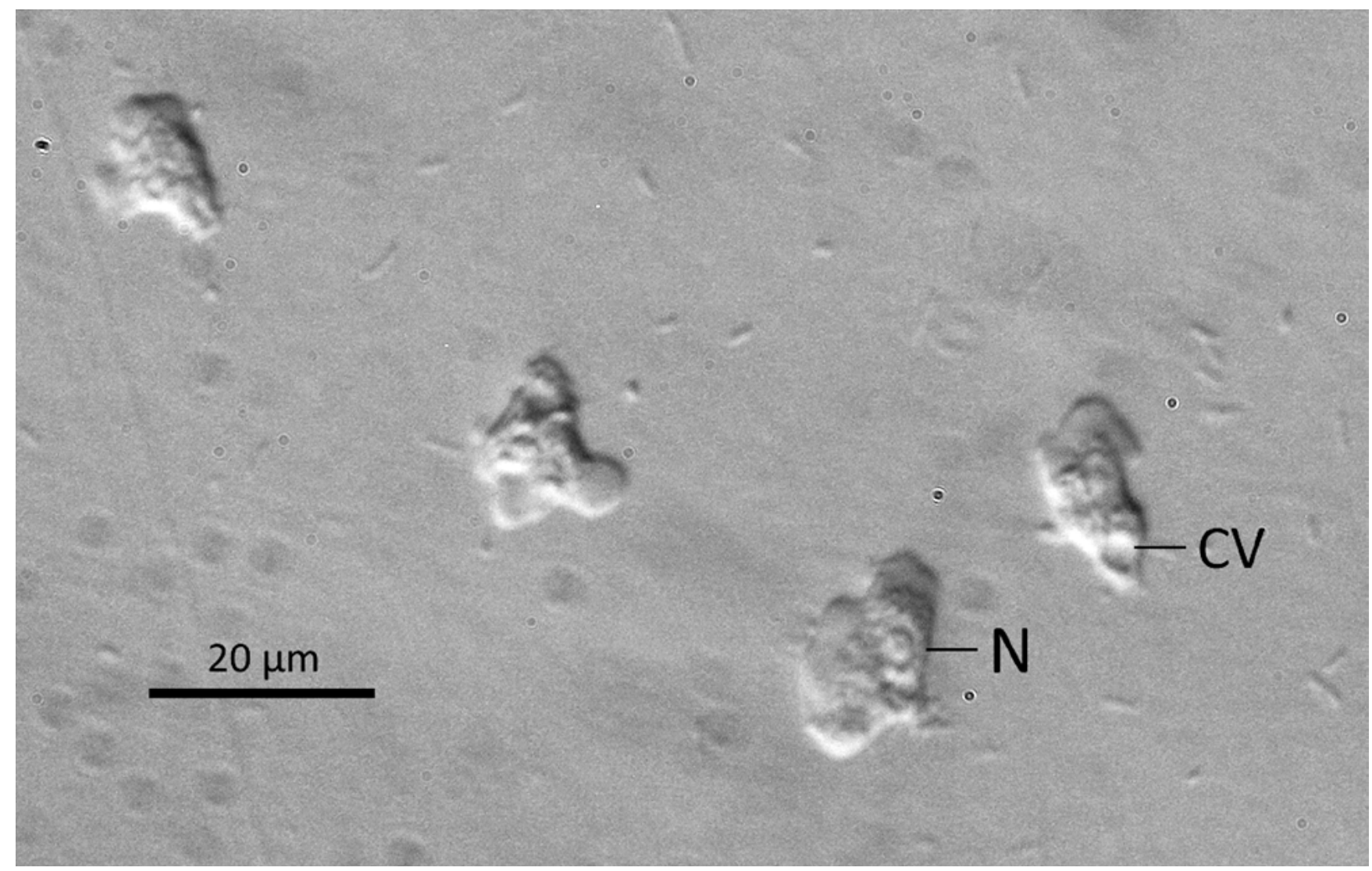

Fig. 1. Trophozoites of Allovahlkampfia minuta showing typical morphology with eruptive pseudopods. Nucleus (N) has a central nucleolus and the contractile vacuole $(\mathrm{CV})$ is typically positioned at the rear of the amoeba. The scale bar is $20 \mu \mathrm{m}$. Food organisms, E. coli are visible in the background.

Fig. 2. Trophozoites gather and encyst on non-nutrient agar plate

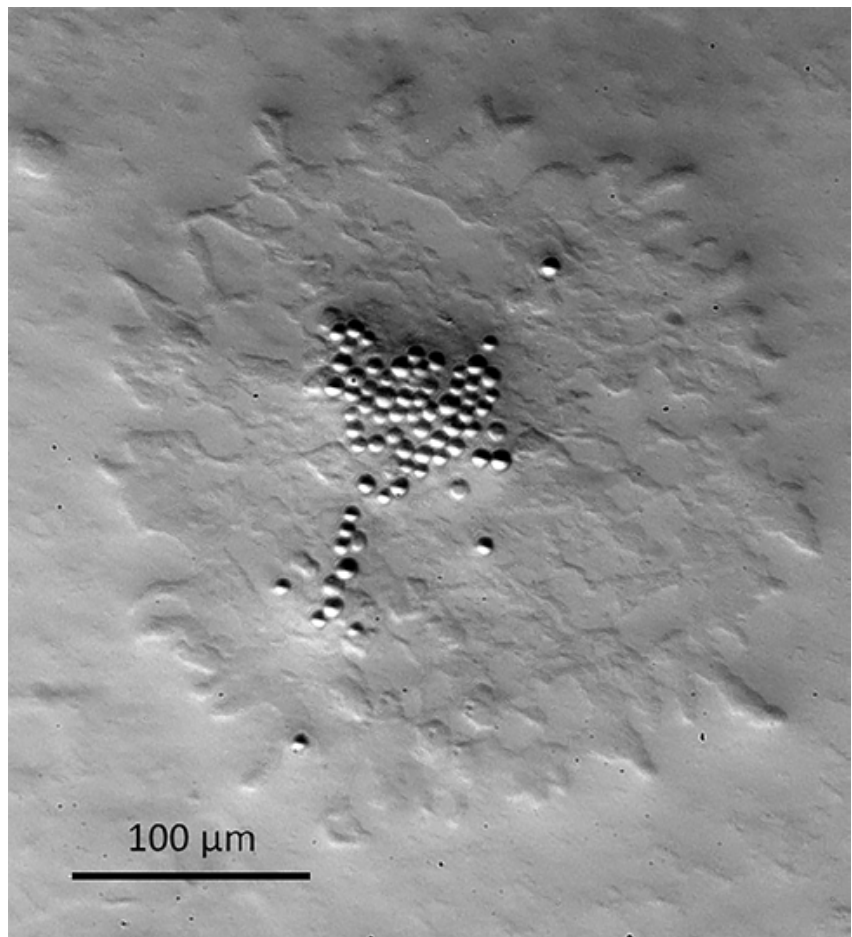
surface as the $E$. coli food or
conditions become less moist. 


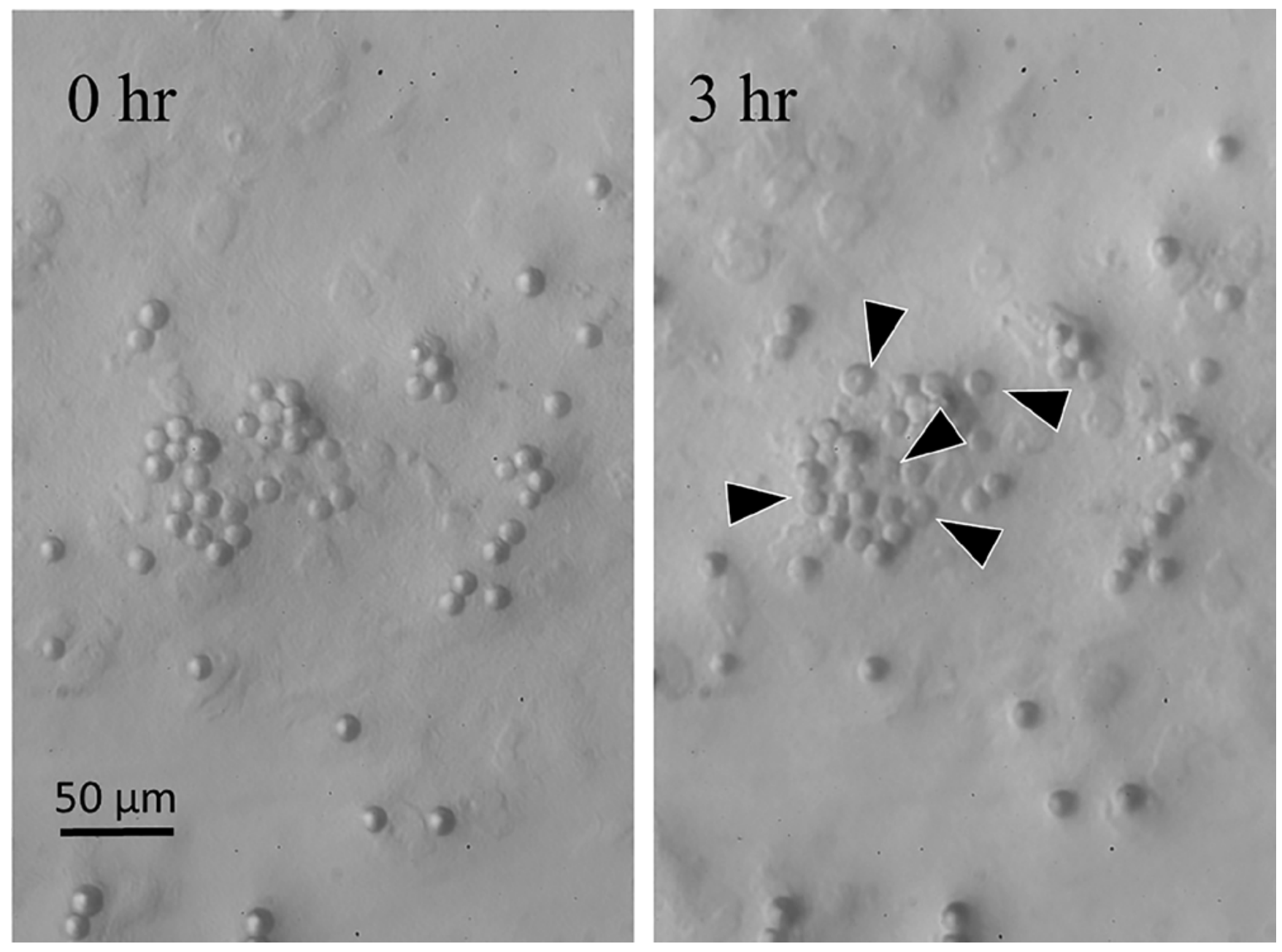

Fig. 3. Cooperative cyst formation. Trophozoites were placed on an agar plate with a monolayer of E. coli. Encystment took place when the plate became dry and the food source locally exhausted. Trophozoites aggregated around the first to encyst, forming a slightly raised mound of cysts. Left panel - This image is the first frame of a video (see supplementary data) taken to follow cyst aggregate formation. Right panel - This is the last image of the same video where black arrowheads point to cysts that have been produced during the time that the video was shot.

and these are very similar in appearance also, especially regarding the tendency to adopt a temporary flabellate locomotory habit. However, these other members of this group are significantly larger (Geisen et al. 2015) (Table 1).

\section{DISCUSSION}

The most notable feature of $A$. minuta is its small size but similarly sized heterolobosean amoebae have been described. For example, Neovahlkampfia nana is $12 \mu \mathrm{m}$ in length (Tyml et al. 2017). Compared to other heteroloboseans including the genus Allovahkmapfia, the locomotion of $A$. minuta seems chaotic and inefficient with frequent direction changes and the production of seemingly immediately redundant pseudopods. A. minuta was observed to adopt a temporal flabellate morphology as is the case for other heteroloboseans for example Heteramoeba clara (Droop 1962) and Vahlkampfia signyensis (Garstecki et al. 2005). The habit of the cysts and trophozoites of $A$. minuta to collect at the meniscus has been reported to be the case with other free-living amoebae (Preston 2003).

The genus Allovahlkampfia is genetically diverse but morphologically uniform and represented so far only by freshwater and soil species. It is not possible to distinguish Allovahlkampfia from several other het- 

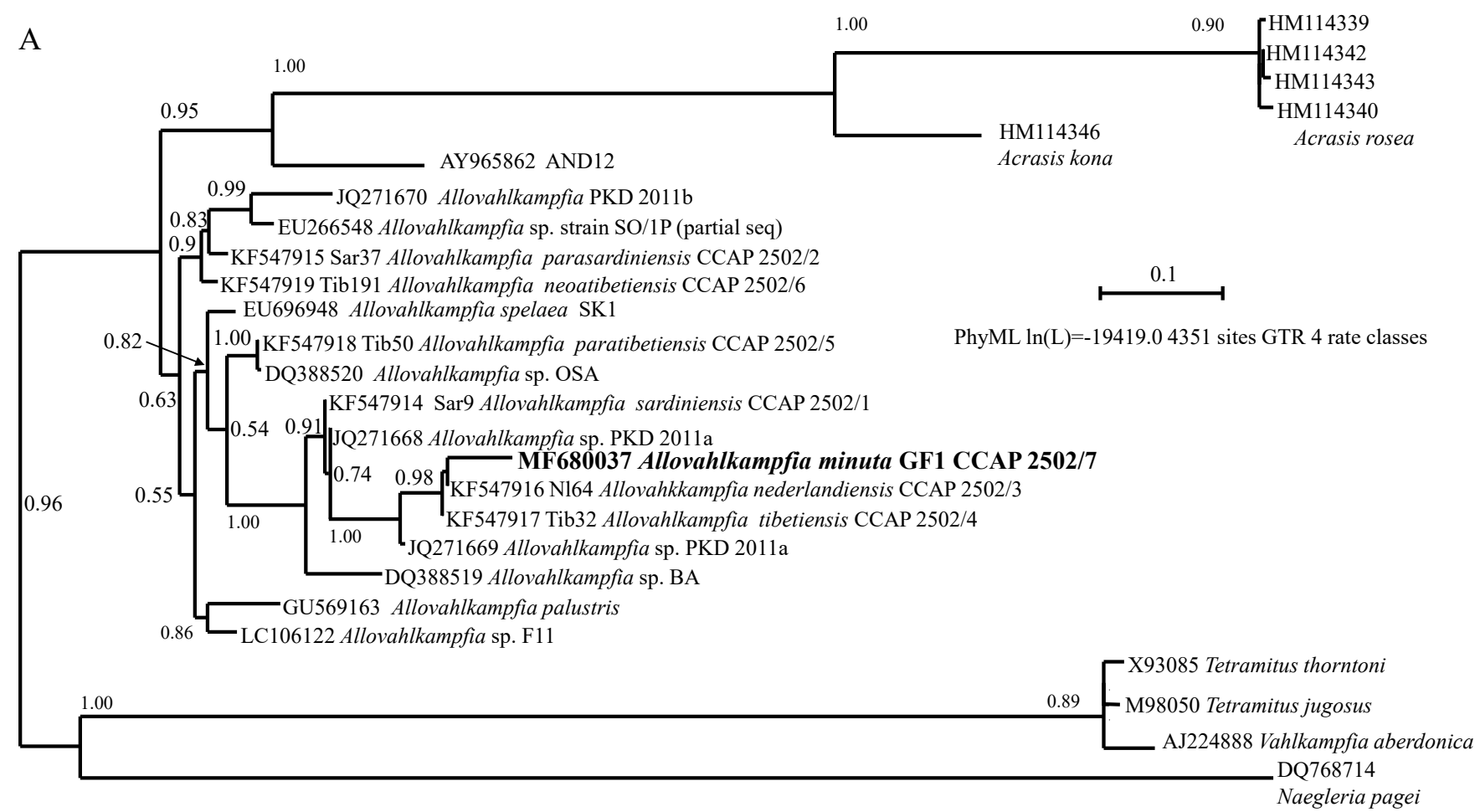

B

PhyML $\ln (\mathrm{L})=-3376.71059$ sites GTR 100 replic. 4 rate classes

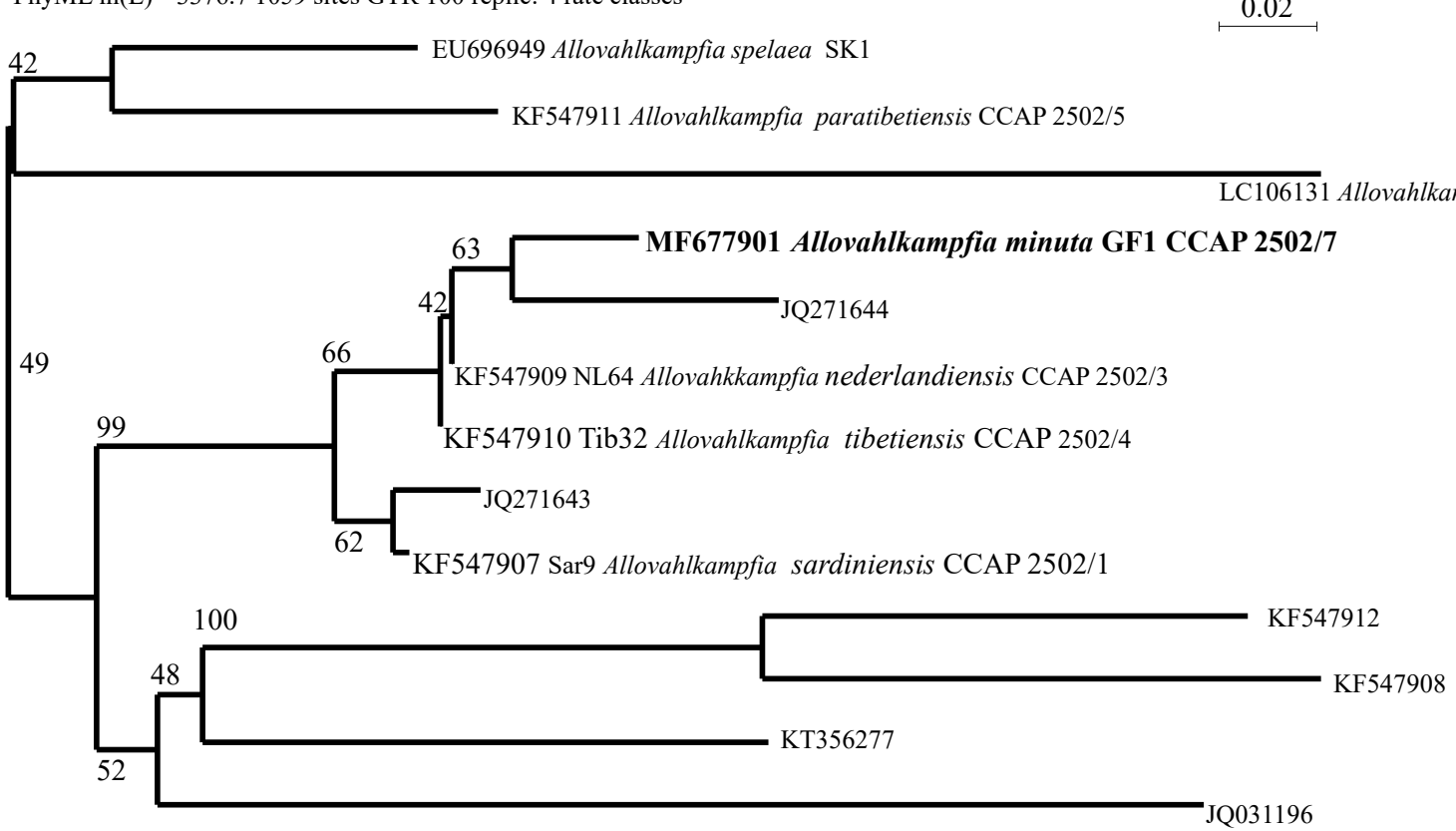

Fig. 4. A. A PhyML phylogenetic tree (GTR model) of members of some heteroloboseans of the genus Allovahlkampfia and Acrasis based on $18 \mathrm{~S}$ rDNA gene. Branch support values at each node indicated as percentages. The GenBank accession code of each sequence is followed by the species names, then strain name (where available). The tree has been rooted using a number of other heterolobosean genera (Tetramitus, Naegleria and Vahlkampfia) as the outgroup. Scale bar represents evolutionary distance. Binomials names for some strains (CCAP 2502/1 to 2502/6) were from the culture collection website (https://www.ccap.ac.uk) as they were not given in the original paper (Geisen et al. 2015). The subject of the present study is highlighted in bold. B. A PhyML phylogenetic tree (GTR model) of members of some heteroloboseans of the genus Allovahlkampfia and Acrasis based on the fragment coding the internal transcribed spacer 1, 5.8S ribosomal RNA, and internal transcribed spacer 2. 
Table 1

\begin{tabular}{lllll}
\hline Species/strain & $\begin{array}{l}\text { Length } \mu \mathrm{m} \\
\text { Average \&/or range }\end{array}$ & $\begin{array}{l}\text { Breadth } \mu \mathrm{m} \\
\text { Average \& range }\end{array}$ & $\begin{array}{l}\text { Cyst diameter } \mu \mathrm{m} \\
\text { Average \&/or range }\end{array}$ & Reference \\
\hline A. palustris & $31(14-43)$ & $6.3(4-12)$ & $8-10$ & Anderson et al. 2011 \\
A. spelaea & $20-40$ & $\mathrm{n} / \mathrm{a}$ & $16-25$ & Walochnik and Mulec, 2009 \\
AND12 & $20-30$ & $\mathrm{n} / \mathrm{a}$ & $\mathrm{n} / \mathrm{a}$ & Lara et al. 2007 \\
A. tibetiensis & $25.2(21.2-31.2)$ & $6.6(5.6-9.2)$ & $6.6(5.0-8.2)$ & Geisen et al. 2015 \\
A. nederlandiensis & $33.2(21.8-50.8)$ & $5.8(3.6-8.6)$ & $6.2(5.0-7.8)$ & Geisen et al. 2015 \\
F11 & 35.8 & 11.4 & 11.5 & Tyml et al. 2016 \\
A. minuta & $13.4(9.5-16.7)$ & $8.1(4.6-15.6)$ & $6.5(4.7-8.9)$ & This study \\
\hline
\end{tabular}

erolobosean genera based on appearance alone (Geisen et al. 2015), even at the level of electron microscopy (González-Robles et al. 2012). The cysts of all presently named species of the genus Allovahlkampfia, including A. minuta described here, lack opercula (Anderson et al. 2011; Walochnik and Mulec 2009; Geisen et al. 2015). This is a feature shared by Acrasis rosea (Hohl and Hamamoto 1969). It is the small size of A. minuta that distinguishes it from others in the genus. None of the previously characterised members of the genus is close in size to the trophozoites or the cysts.

Since reports of morphological similarities between vahlkampfids and acrasids were published (Page 1978; Page and Blanton 1985), several authors have reported a close genetic affinity also (Keeling and Doolittle 1996; Roger et al. 1996). Comparing ribosomal genes allowed Allovahlkampfia and Acrasis to be placed in clade "Acrasidia" within the Tetramitia (De Jonckheere et al. 2011; Harding et al. 2013; Geisen et al. 2015; Pánek et al. 2017). In this connection, the Allovahlkampfid strain AND12 (Lara et al. 2007) is especially interesting as it is currently the most closely known relative of the genus Acrasis. However, it was only partially characterized and because it was observed in liquid culture, its ability to develop fruiting bodies is untested (Lara et al. 2007). Unfortunately, this strain is no longer available in culture (Ekelund, Pers. Comm), but amoebae at the boundary between Allovahlkampfia and Acrasis may reveal details on the development of sporogenesis.

The aggregation of $A$. minuta trophozoites during encystment, produces images very similar to those seen in the acrasids especially the morphologically simple Acrasis helenhemmesae (see figures 14-16 in Brown et al. 2010). This tendency to aggregate may be shared with others of the genus as $A$. palustris and $A$. spelaea cysts are also reported to occur in clumps, although no further information about this was given (Anderson et al. 2011; Tolba et al. 2016). The significance of this aggregation is not yet clear but we have found time lapse video evidence (see supplementary data) for chemotaxis towards the encysting centre. There may be increased survival of aggregated cysts, or the aggregation may, like is this case in the acrasids be a prelude to the production of Allovahlkampfid fruiting bodies that may form under as-yet unknown circumstances. In this regard, it is interesting to note that a simple linear fruiting body has been observed in Allovahlkampfia strain BA (Brown et al. 2012), however this was observed on only one occasion. Other hints that Allovahlkampfia can produce multicellular aggregates such as fruiting exist. The isolate, Allovahlkampfia strain OSA, has been described as "an orange stalk-like fungal growth habit" (Shutt 2006) and while this description is a little vague, it is compatible with it having a multi-cellular or at least aggregative stage. Confusingly however, neither BA nor OSA formed fruiting bodies according to the worker who isolated them (Schutt 2006). Strains BA and OSA were assigned to A. spelaea (Brown et al. 2012) but this suggestion has not been adopted by later workers (Geisen et al. 2015) and is not supported by our $18 \mathrm{~S}$ rDNA analysis. The aggregation that we observed with encysting $A$. minuta amoeba is superficially similar to the aggregation during the encystment of the amoebozoan, Vannella pentlandii (Maciver et al. 2017). Also, the closely related Vannella fimicola produces an actual fruiting body (Olive, 1962). The ability to fruit has evolved apparently independently, in other amoebozoan groups, including Luapeleamoeba hula within family Acanthamoebidae (Shadwick et al. 2016), and surprisingly, including the genus Acanthamoeba itself (Tice et al. 2016). Amoebae other than the Amoebozoa 
are also known to produce fruiting bodies (Kang et al. 2017). There is little evidence of sexual recombination in the acrasids (Brown et al. 2012), but it is likely that the purpose of these fruiting bodies in the acrasids may be a dispersive strategy carrying the spores off the substrate to be carried by air currents, or passing birds and insects.

Many genera of free living amoeba contain species pathogenic to humans and while most of these including Acanthamoeba, Balamuthia and Sappinia are members of the amoebozoa some are heteroloboseans. Acanthamoeba is notorious for causing Acanthamoeba keratitis (Lorenzo-Morales et al. 2015), a sight threatening and extremely painful infection of the eye but there are reported cases of a similar keratitis associated with the presence of amoebae tentatively identified as being Vahlkampfia but in the presence of other organisms (Aitken et al.1996; Alexandrakis et al. 1998; Niyyati et al. 2010; González-Robles et al. 2012). These reports, while not concluding a directly causal link, do reveal that that heteroloboseans can survive on the human eye. Stronger evidence suggests that an amoeba $\mathrm{SO} / 1 \mathrm{P}$ now known to belong in genus Allovahlkampfia (Fig. 2), cause keratitis in humans with herpes virus (Ozkoc et al. 2008) and that this same strain produced a similar infection in rat models (Huseein et al. 2016) (presumably in the absence of herpes virus since it was not mentioned). A second case where an amoeba closely related to Allovahlkampfia spelaea was reported to cause keratitis has also been reported (Tolba et al. 2016) and this strain also caused keratitis in a rabbit model. Whereas $93 \%$ of Acanthamoeba keratitis cases are associated with contact lens use (Radford et al. 1998), the few cases reported for heterolobosean amoebae have been dominated by trauma to the eye surface (Alexandrakis et al. 1998; Ozkoc et al. 2008; Tolba et al. 2016). It is firmly established that another heterolobosean, Naegleria fowleri causes the usually fatal brain infection primary amoebic meningoencephalitis in humans (Siddiqui et al. 2016). The finding that $A$. minuta does not grow above $28^{\circ} \mathrm{C}$, and its failure to cope with elevated osmolarity, would seem to rule it out as a potential human eye pathogen where the temperature varies between 32 and $36^{\circ} \mathrm{C}$ (Purslow et al. 2005).

\section{Allovahlkampfia minuta n. sp.}

Description. The locomotive morphology of this species is typical for small heterolobosean amoebae. Amoeba length $13.4 \mu \mathrm{m}$ (range 9.5-16.7) and width $8.1 \mu \mathrm{m}$ (range 4.6-15.6). Length:breadth ratio is 1.65 .
A well-developed uroid is not often visible but trailing uroidal filaments are occasionally observed on glass and tissue culture plastic. The spherical nucleus is variable in diameter but it ranges between 1.5-2.5 $\mu \mathrm{m}$. There is a central nucleolus. A contractile vacuole is usually visible in trophozoites in Neff's saline or distilled water. This is usually located at the rear of the amoeba, often within the bulbous uroid, if it is present. Growth on E. coli is observed between 4 and $28^{\circ} \mathrm{C}$, with an optimum at $24^{\circ} \mathrm{C}$. The pelleted trophozoites have a distinct pink/orange colour.

Cysts. Spheroidal diameter $6.5 \mu \mathrm{m}$ (range 4.7-8.9) with a single cell wall and lacking pores. The mononuclear cysts are usually formed in aggregates.

Etymology. This isolate is significantly smaller than any other described Allovahlkampfia species, hence the name "minuta".

Type locality. Bank of Loch Shiel (freshwater) near the Glennfinnan monument (latitude 56.8687; longitude -5.4378 ), Scotland, UK. The elevation is $4 \mathrm{~m}$.

Habitat. Alluvial soil.

Type material. A culture of $A$. minuta has been deposited with the Culture Collection of Algae and Protists, accession number CCAP 2502/7, and gene sequences deposited with GenBank, MF680037 and MF677901 for the $18 \mathrm{~S}$ rRNA gene and the ITS region respectively.

Acknowedgements. We are grateful to Mr Samuel Casasola Zamora for collecting soil samples. We are pleased to acknowledge support from Mexico's National Council of Science and Technology (CONACYT) and a University of Edinburgh College of Medicine and Veterinary Medicine PhD Studentship. Support from Amoebics Ltd, Edinburgh is also acknowledged. We also gratefully acknowledge two anonymous reviewers for their useful and insightful comments on an earlier version of the manuscript.

\section{REFERENCES}

Aitken D., Hay J., Kinnear F. B., Kirkness C. M., Lee W. R., Seal D.V. (1996) Amebic keratitis in a wearer of disposable contact lenses due to a mixed Vahlkampfia and Hartmanella infection. Ophthalmol.103: 485-494

Anderson O. R., Wang W. E. N., Faucher S. P., Bi K., Shuman H. A. (2011) A new heterolobosean amoeba Solumitrus palustris n. g., n. sp. isolated from freshwater marsh soil. J. Eukaryot. Microbiol. 58: $60-67$

Alexandrakis G., Miller D., Huang A. J. W. (1998) Amebic keratitis due to Vahlkampfia infection following corneal trauma. Arch. Ophthalmol. 117: 950-951

Brown S., De Jonckheere J. F. (1999) A reevaluation of the amoeba genus Vahlkampfia based on SSUrDNA sequences. Eur. J. Protistol. 35: 49-54

Brown M. W., Silberman J. D., Spiegel F. W. (2010) A morphologically simple species of Acrasis (Heterolobosea, Excavata), Acrasis helenhemmesae n. sp. J. Eukaryot. Microbiol. 57: 346-353 
Brown M. W., Silberman J. D., Spiegel F. W. (2012) A contemporary evaluation of the acrasids (Acrasidae, Heterolobosea, Excavata). Eur. J. Protistol. 48: 103-123

Carter R. F. (1970) Description of a Naegleria species isolated from two cases of primary amoebic meningoencephalitis and of the experimental pathological changes induced by it. J. Pathol. 100: $217-244$

De Jonckheere J. F., Murase J., Opperdoes F. R. (2011) A new thermophilic heterolobosean amoeba, Fumarolamoeba ceborucoi, gen. nov., sp. nov., isolated near a fumarole at a volcano in Mexico. Acta Protozool. 50: 41-48

Droop M. R. (1962) Heteramoeba clara n. gen., n. sp., a sexual biphasic amoeba. Arch. Microbiol. 42: 254-266

Garstecki T., Brown S., De Jonckheere J. F. (2005) Description of Vahlkampfia signyensis n. sp. (Heterolobosea), based on morphological, ultrastructural and molecular characteristics. Eur. J. Protistol. 41: 119-127

Geisen S., Bonkowski M., Zhang J., De Jonckheere J. F. (2015) Heterogeneity in the genus Allovahlkampfia and the description of the new genus Parafumarolamoeba (Vahlkampfiidae; Heterolobosea). Eur. J. Protistol. 51: 335-349

González-Robles A., Salazar-Villatoro L., González-Lázaro M., Omaña-Molina M., Martínez-Palomo A. (2012) Vahlkampfia sp: Structural observations of cultured trophozoites. Exp. Parasitol. 130: 86-90

Gouy M., Guindon S., Gascuel O. (2010) SeaView Version 4: A multiplatform graphical user interface for sequence alignment and phylogenetic tree building. Mol. Biol. Evol. 27: 221-224

Guindon S., Gascuel O., (2003) A simple, fast, and accurate algorithm to estimate large phylogenies by maximum likelihood. Syst. Biol. 52: 696-704

Hall T. A., (1999) BioEdit: a user-friendly biological sequence alignment editor and analysis program for Windows 95/98/NT. Nucl. Acids Symp. Ser. 41: 95-98

Harding T., Brown M. W., Plotnikov A., Selivanova E., Park J. S., Gunderson J. H., Baumgartner M., Silberman J. D., Roger A. J., Simpson A. G. (2013) Amoeba stages in the deepest branching heteroloboseans, including Pharyngomonas: evolutionary and systematic implications. Protist 164: 272-286

Hohl H. R., Hamamoto S. T. (1969) Ultrastructure of Acrasis rosea, a cellular slime mold, during development. J. Protozool. 16: $333-344$

Huseein E. A. M., Anwer F. E. S., Abed G. H., Abdel-Gahfar K. S., Omar H. E. D. M. (2016) Keratitis induced by Allovahlkampfia spelaea in experimental rat model: a trial for treatment with ellagic acid. Eur. J. Biol. Res. 6: 299-309

Kang S., Tice A. K., Spiegel F. W., Silberman J. D., Pánek T., Čepička I., Kostka M., Kosakyan A., Alcântara D. M., Roger A. J., Shadwick L. L., Smirnov A., Kudryavstev A., Lahr D. J. G., Brown M. W. (2017) Between a pod and a hard test: the deep evolution of amoebae. Mol. Biol.Evol. 34: 2258-2270

Keeling P. J., Doolittle W. F. (1996) Alpha-tubulin from early diverging eukaryotic lineages and the evolution of the tubulin family. Mol. Biol. Evol. 13: 1297-1305

Khan N. A., Jarroll E. J., Paget T. A. (2001) Acanthamoeba can be differentiated by the polymerase chain reaction and simple plating assays. Curr. Microbiol. 43: 204-208

Lara E., Berney C., Ekelund F., Harms H., Chatzinotas A. (2007) Molecular comparison of cultivable protozoa from a pristine and a polycyclic aromatic hydrocarbon polluted site. Soil Biol. Biochem. 39: 139-148
Lorenzo-Morales J., Lindo J. F., Martinez E., Calder D., Figueruelo E., Valladares B., Ortega-Rivas A. (2005) Pathogenic Acanthamoeba strains from water sources in Jamaica, West Indies. Ann. Trop. Med. \& Parasitol. 99: 751-758

Lorenzo-Morales J., Maciver S. K. (2006) Long-term, low maintenance storage of Acanthamoeba strains. Parasitology Research 99: $743-745$

Lorenzo-Morales J., Khan N. A., Walochnik J. (2015) An update on Acanthamoeba keratitis: diagnosis, pathogenesis and treatment. Parasite 22: 10-20

Maciver S. K., De Obeso Fernandez Del Valle A., Koutsogiannis Z. (2017) Vannella pentlandii n. sp., (Amoebozoa, Discosea, Vannellida) a small, cyst-forming soil amoeba. Exp. Parasitol. (in press)

Mohamed M. E., Huseein E. A., Farrag H. M., Mostafa F. A. A., Hassan A. T. (2016) Allovahlkampfia spelaea is a potential environmental host for pathogenic bacteria. J. Bacteriol. Parasitol. 7: $255-262$

Niyyati M., Lorenzo-Morales J., Rezaie S., Rahimi F., MartínNavarro C. M., Mohebali M., Rezaeian M. (2010) First report of a mixed infection due to Acanthamoeba genotype T3 and Vahlkampfia in a cosmetic soft contact lens wearer in Iran. Exp. Parasitol. 126: 89-90

Olive L. S. (1962) The genus Protostelium. Amer. J. Botany 49: 297-303

Ozkoc S., Tuncay S., Delibas S. B., Akisu C., Ozbek Z., Durak I., Walochnik J. (2008) Identification of Acanthamoeba genotype T4 and Paravahlkampfia sp. from two clinical samples. J. Med. Microbiol. 57: 392-396

Page F. C. (1976) An illustrated key to freshwater and soil amoebae. Freshwater Biological Association, Ambleside, England.

Page F. C. (1978) Acrasis rosea and the possible relationship between Acrasida and Schizopyrenida. Archiv für Protistenkunde. 120: $169-181$

Page F. C., Blanton R. L. (1985) The Heterolobosea (Sarcodina:Rhizopoda), a new class uniting the Schizopyrenida and the Acrasidae (Acrasida). Protistologica 21: 121-132

Pánek T., Čepička I. (2016) Diversity of Heterolobosea. In "Genetic Diversity of Microorganisms", 3-26. Eds. Caliskan M., Rijeka $\mathrm{M}:$ InTech.

Pánek T., Simpson A. G. B., Brown M. W., Dyer B. D. (2017) Heterolobosea in "Handboook of the Protists" Archibald et al., eds Springer Int. Publ.

Perkins D. L., Jahn T. L. (1970) Amoeboflagellate transformations and the Gibbs-Donnan ratio. J. Protozool. 17: 168-172

Preston T. M. (2003) The water-air interface: a microhabitat for amoebae. Eur. J. Protistol. 39: 385-389

Purslow C., Wolffsohm J. S., Santodomingo-Rubido J. (2005) The effect of contact lens wear on dynamic ocular surface temperature. Contact Lens \& Ant. Eye 28: 29-36

Radford C. F., Lehmann O. J., Dart J. K. (1998) Acanthamoeba keratitis: multicentre survey in England 1992-1996. Br. J. Ophthalmol. 82: 1387-1392

Roger A. J., Smith M. W., Doolittle R. F., Doolittle W. F. (1996) Evidence for the heterolobosea from phylogenetic analysis of genes encoding glyceraldehyde-3-phosphate dehydrogenase. $J$. Euk. Microbiol. 43: 475-485

Shadwick L. L., Brown M. W., Tice A. K., Spiegel F. W. (2016) A new amoeba with Protosteloid Fruiting: Luapeleamoeba hula n. g. n. sp. (Acanthamoebidae, Centramoebida, Amoebozoa). Acta Protozool. 55: 123-134 
Shutt T. E. (2006) Origins and evolution of mitochondrial transcription and replication machinery. Ph.D. Thesis, Dalhousie University, Halifax, Nova Scotia.

Siddiqui R., Ali K. M., Cope J. R., Khan N. A. (2016) Biology and pathogenesis of Naegleria fowleri. Acta Tropica 164: 375-394

Tice A. K., Shadwick L. L., Fiore-Donno A. M., Geisen S., Kang S.,Schuler G. A.,Spiegel F. W., Wilkinson K. A., Bonkowski M., Dumack K., Lahr D. J. G., Voelcker E., Clauß S., Zhang J. Brown M. W. (2016) Expansion of the molecular and morphological diversity of Acanthamoebidae (Centramoebida, Amoebozoa) and identification of a novel life cycle type within the group. Biology Direct 11: 69; doi 10.1186/s13062-016-0171-0

Tolba M. E. M., Huseein E. A. M., Farrag H. M. M., Mohamed H. E. D., Kobayashi S., Suzuki J., Sugano S. (2016) Allovahlkampfia spelaea causing keratitis in humans. PLoS Negl. Trop. Dis. 10: e0004841
Tyml T., Skulinová K., Kavan J., Ditrich O., Kostka M., Dyková I. (2016) Heterolobosean amoebae from Arctic and Antarctic extremes: 18 novel strains of Allovahlkampfia, Vahlkampfia and Naegleria. Eur. J. Protistol. 56: 119-133

Tyml T., Lares-Jiménez L. F., Kostka M., Dyková I. (2017) Neovahlkampfia nana $\mathrm{n}$. sp. Reinforcing an underrepresented subclade of Tetramitia, Heterolobosea. J. Euk. Microbiol. 64: 78-87

Walochnik J., Mulec, J. (2009) Free-living amoebae in carbonate precipitating microhabitats of karst caves and a new vahlkampfiid amoeba, Allovahlkampfia spelaea gen. nov., sp. nov. Acta Protozool. 48: 25-33

Received on $10^{\text {th }}$ August, 2017; revised on $3^{\text {rd }}$ October, 2017; accepted on $8^{\text {th }}$ October, 2017

\section{Supplementary data available on journal website}

Video 1. Locomotion of Allovahlkampfia minuta trophozoites. Real time video micrography shows typical eruptive locomotion but also show the chaotic nature of the progression of this species.

Video 2. Cyst chemotaxis. Time lapse video with frames every 15 seconds, total time 3 hours. While some trophozoites continue to move feed and divide, others are drawn toward existing cysts and they too then encyst. Contractile vacuole activity gradually lessens as cyst development takes place. 